\title{
Timing performances of a data acquisition system for Time of Flight PET
}

\author{
Matteo Morrocchi , Sara Marcatili , Nicola Belcari , Maria G. Bisogni , Gianmaria Collazuol , \\ Giovanni Ambrosi , Francesco Corsi , Maurizio Foresta ,Cristoforo Marzocca , Gianvito Matarrese , \\ Giancarlo Sportelli , Pedro Guerra , Andres Santos , Alberto Del Guerra
}

\begin{abstract}
A B S T R A C T
We are investigating the performances of a data acquisition system for Time of Flight PET, based on LYSO crystal slabs and 64 channels Silicon Photomultipliers matrices $\left(1.2 \mathrm{~cm}^{2}\right.$ of active area each). Measurements have been performed to test the tining capability of the detection system (SirM matices coupled to a LYSO slab and the read-out electronics) with both test signal and radioactive source.
\end{abstract}

\section{Introduction}

A new acquisition system based on SiPM matrices and LYSO crystals PET is under development at the Department of Physics, University of Pisa and INFN Pisa.

Silicon Pholo Multipliers are suitable for PET application due to their high gain and low noise level; an important feature of the SiPMs is their lugh time resolution that could be exploited in the so-called Time of Flight (TOF) PET, a technique useful to improve the system sensitivity and the signal to noise ratio of the PET images.

Good timing performance is presently achieved with PbF2 crystals and micro-channel plates photodetectors [1] with the counterpart of a very low efficiency.

A small prototype composed of two $8 \times 8$ SiPM matrices, LYSO crystal and a custom data acquisition system is under studies for further development of a smakl animal high resolution PET scanner.

The aim of this work is to investigate the time resolution capability of our system in view of a possible application to clinical TOF-PET. However our trigger set-up has not been optimized for high time resolution, because Time of Flight technique is of limited advantage in small animal imaging, that is our primary goal.
The acquisition system is composed of two detection heads, each one equipped with 64 acquisition channels and read-out by dedicated Front-Fnd ASICs and Data Acquisition boards (DAQ board5). The present version of the detection system has been tested to assess its timing performances using both radioactive sources and test signals; these have been useful to evaluate the contribution of the electronics to the timing performances of the whole system.

\section{Materials and methods}

The detector device consists of two $8 \times 8$ SiPM matrices with $1.5 \mathrm{~mm}$ pitch. The pixel size is $1.5 \times 1.4 \mathrm{~mm}^{2}$ for a total of 840 microcells of $50 \times 50 \mu \mathrm{m}^{2}$ size. A common bias voltage is applied to the whole matrix. Two LYSO slabs, $12 \times 12 \times 5 \mathrm{~mm}^{3}$ each, are coupled to two SiPM matrices in PET configuration. Optical grease is used to improve the optical matching and crystals are wrapped with teflon to increase the amount of light collected. The crystal is fixed on to the detector with custom holders in order to avoid mismatching between scintillator and matrix.

The acquisition system is composed of a motherboard handling up to 18 DAQ boards. Fach DAQ board hosts a custom 8-channels Front-End ASIC $[2,3]$ (named BASIC). The inpul stage or each BASICs channel is split into two branches: a fast one made of a curtent buffer connected to a leading edge discriminator for a fast trigger generation; a slow one made of a charge preamplifier connected to a peak detector. The eight outputs are multiplexed 
and sent to a 10 bits ADC hosted on each DAQ board. Data from all the ADCs are handled by an FPGA located on the motherboard, then sent to a host PC through a USB port.

Two DAQ boards with 8 channels each are already available and others are under tests.

Twelve different trigger levels could be chosen and the time distribution of the digital trigger signals between the two DAQ has been analyzed.

\subsection{Electronic's timing contribution}

Preliminary measurements have been performed to estimate the intrinsic time jitter of the Front-End electronics; test signals were used to simulate different charge injections from the SiPMs.

To perform this measurement, a synchronous test signal has been sent to the input of two BASICs, and then the time delay of the two fast-OR signals has been acquired. Signals of the same amplitude were sent to both boards; thus any signal time walk contribution is not affecting the measurement. Tests signals consisting of square waves of different amplitudes generated by a pulse generator LeCroy9210 are sent to a shaper, whose circuit is represented in Fig. 1.

For charge injection we have used a capacitance of $390 \mathrm{pF}$ and a partition circuit consisting of two resistances to reproduce the real amplitude of the signal. The signal obtained has a rise time of the order of a few nano-seconds and a fall time of about $13 \mathrm{~ns}$, that well reproduces the SiPM signal shape. For the input impedance of the Front-End circuit a value of $20 \Omega$ was chosen; this value was given as upper limit for the buffer current circuit in the ASIC design.

The two digital signals of Fast-Or trigger have both been sent to a LeCroy LC684DM scope to evaluate the time distribution.

The scope contribution to the timing distribution has been previously measured with synchronous square waves and quantified to less than $40 \mathrm{ps}$.

\subsection{Timing measurements}

To measure the time jitter of the whole detection system, the two sensors have been mounted one in front of the other and both irradiated with a $\mathrm{Na}-22$ source.

The 8 central channels of each SiPM matrix in a row have been connected to the input channels of the DAQ board and the trigger of both BASICs have been sent to the scope to evaluate the time delay between the two digital signals. The bias voltage of the two matrices has been chosen so as to have the same gain in both detectors. A schematic drawing of the acquisition set-up is shown in Fig. 2.

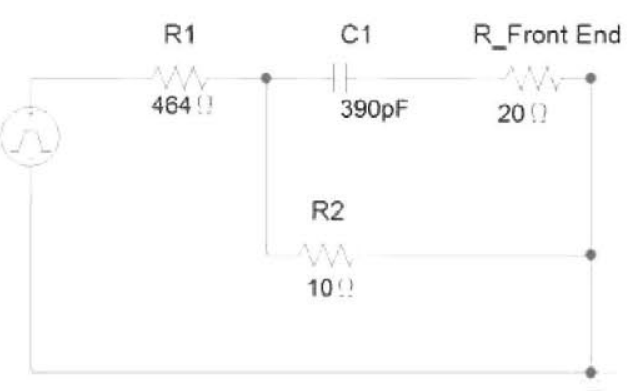

Fig. 1. Schematic of the charge injection circuit where the $20 \Omega$ impedance symbolizes the Front-End contribution.

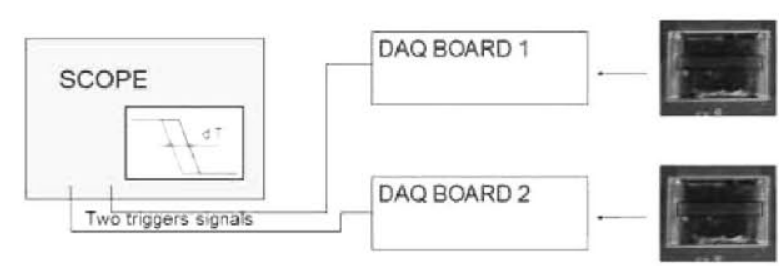

Fig. 2. Scheme of the set-up used to evaluate the time coincidence distribution.

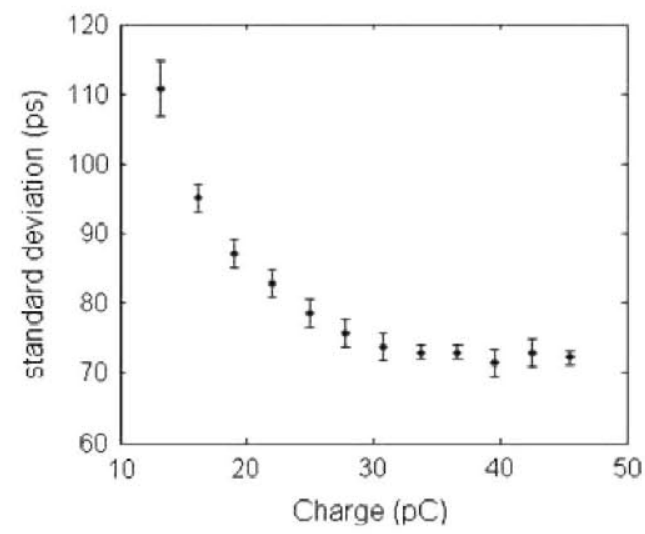

Fig. 3. Standard deviation of the time delay distribution as a function of the charge injected.

\section{Results}

The standard deviation of the time delay distribution as a function of the charge injected with test signals is shown in Fig. 3.

The charge input has been quantified using the multiplexer output. The voltage at the output of the pulse generator was properly chosen so as to work inside the dynamic range of the CSA.

As the charge injected increases, the time jitter decreases due to the improvement of the signal to noise ratio. The minimum value achieved is $70 \mathrm{ps}$ sigma.

Time delay distribution could also be convoluted with a Na-22 spectrum acquired in time coincidence to evaluate only the electronic contribution over the entire range of events with a result lower than $100 \mathrm{ps}$.

Timing measurement with SiPM matrices was performed using the lower possible threshold and without selecting any energy window for the events.

Results shown below do not represent the best possible performance of the system because just a single threshold only is used to trigger the events.

A high SiPM's operating voltage was chosen (working inside the dynamic range of the DAQ as in standard acquisition) to perform the best possible result, according to the previous measurements. The time delay distribution is shown in Fig. 4.

The large time jitter of the signals ( $490 \mathrm{ps}$ sigma) mainly derives from the time walk among signals of different amplitude, and strictly depends on the adopted trigger method.

In fact, with the present front-end architecture, only one threshold can be chosen for energy selection. An improvement of the Front-End foresees a double discriminator architecture in which a very low threshold optimizes timing performances while a second threshold allows for energy selection, thus reducing the time jitter.

Previous measurements executed with a double threshold method show a standard deviation of less than $150 \mathrm{ps}$ for the photodetector-crystal contribution in timing coincidence; thus 


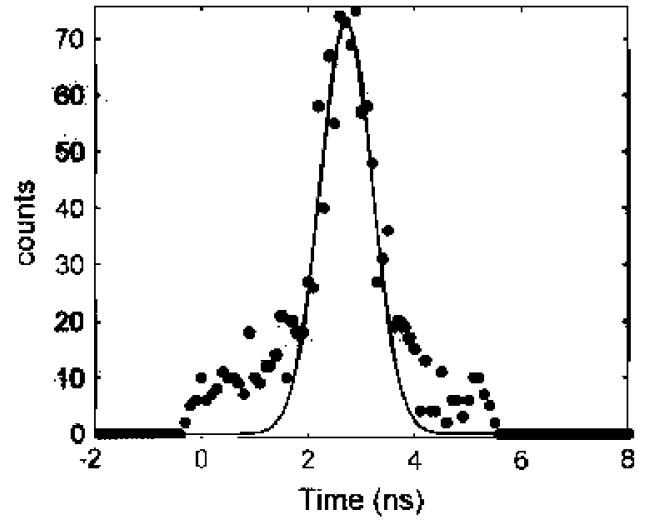

Fig 4. Time delay distribution becween two central rows of two differene SiPMs matrices, each contspled with al LYSO crystal slab.

a total jitter of less than $200 \mathrm{ps}(s$ ) is expected for the whole system with an implementation of the optimal trigger capabilities.

\section{Conclusions}

The time performance of the electronic acquisition system was tested with promising Jesufts for Time Or Flight PET application.

Measurements taken with simulated test signals give $70 \mathrm{ps}$ standard deviation for the intrinsic electronic jitter with a signal amplitude comparable with photo-peak events.
Time distribution obtained with SiPM matrices is dominated by the contribution of the time walk due to signals of different energies. Currently, the single threshold architecture limits the overall performance of the system because a double threshold trigger level is necessary to maximize the timing capabilities.

Combining the results for the electronic jitter with previous coincidence measurements, obtained at the scope selecting only the photo-peak events with LSO:Ce,Ca crystals |4] with the double threshold method, we expect a standard deviation of less than $200 \mathrm{ps}$ for the system at $511 \mathrm{keV}$.

\section{Acknowledgment}

This work is partially supported by FP7-Envision project funded by EU Seven Framework G. Sportelli, P. Guerra and A Santos want to acknowledge partial financial support from Spain's Science Ministry (projects CDTI AMIT and TEC201128972-C02-02) and European Regional Development Funds.

\section{References}

1) Dolenter, et al., Time-of-Flight PFT using Cherenkov Photons Produced in PbF2 Crystals, Piresentation al NDIP2011.

[2] S. M,urcalili, et al. Nuclear Instruments and Methods in Physics Research A 659 (2011) 494 .

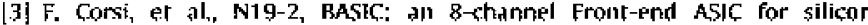
photomultiplice detectors 2009 IEEE Nuclear Scicnce Symposium Conference Record 2009, op. 1082-1087.

[4i M.G. Bisogni. G.M. Collazuol, S. Marcatili, C.L. Malcher, $\Lambda$ Dej Guera, Nuclea Instuments and Methods ìn Physics Research Section $A 028$ ( 1 ) (2011) 423. 